\title{
Cosmic Ray Modulation Observed by the Princess Sirindhorn Neutron Monitor at High Rigidity Cutoff
}

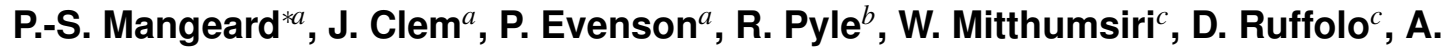 \\ Sáiz $^{c}$, T. Nutaro ${ }^{d}$ \\ ${ }^{a}$ Bartol Research Institute, University of Delaware, Newark, DE 19716, USA. \\ ${ }^{b}$ Pyle Consulting Group, Inc., St. Charles, IL 60174, USA. \\ ${ }^{c}$ Department of Physics, Faculty of Science, Mahidol University, Bangkok 10400, Thailand. \\ ${ }^{d}$ Department of Physics, Faculty of Science, Ubon Ratchathani University, Ubon Ratchathani \\ 34190, Thailand. \\ E-mail: psmangeardegmail.com, clemebartol.udel.edu, evenson@udel.edu, \\ rogerpyle@gmail.com, warit.mit@mahidol.ac.th, \\ david.ruf@mahidol.ac.th, alejandro.sai@mahidol.ac.th, \\ tnutaro@yahoo.com.
}

Solar modulation refers to Galactic cosmic ray (GCR) variations with the $\sim 11$-year sunspot cycle and $\sim 22$-year solar magnetic cycle, and is relevant to the space radiation environment and effects on Earth's atmosphere. Its complicated dependence on solar and heliospheric conditions is only roughly understood but has been empirically modeled in terms of a single modulation parameter. Most analyses of solar modulation used neutron monitor (NM) data from locations with relatively low geomagnetic cutoff rigidity, i.e., the threshold for cosmic rays to penetrate Earth's magnetic field. The Princess Sirindhorn Neutron Monitor (PSNM) at Doi Inthanon, Thailand has the highest cutoff rigidity $(\approx 17 \mathrm{GV}$ ) where observations span a complete solar modulation cycle (since late 2007). The pattern of solar modulation at Doi Inthanon during 2011-2014 was qualitatively very different from that at low geomagnetic cutoff, and is not well described by the same modulation parameter. At other times, NM count rates from Doi Inthanon and McMurdo, Antarctica (cutoff $<0.01 \mathrm{GV}$ ) are linearly correlated and confirm the observation from latitude surveys in the previous solar cycle that the slope of the correlation changes with solar magnetic polarity. Low solar magnetic tilt angles $\left(<40^{\circ}\right.$, at negative polarity) were well correlated with variations at both NM stations as expected from drift models. At higher tilt angle, the Doi Inthanon count rate is well correlated with the interplanetary magnetic field, which is consistent with an expected increase in diffusion at high rigidity short-circuiting effects of drifts and the heliospheric current sheet.

35th International Cosmic Ray Conference

10-20 July, 2017

Bexco, Busan, Korea

${ }^{*}$ Speaker. 


\section{Introduction}

Galactic cosmic rays (GCRs) are sensitive to solar and heliospheric effects while they propagate from outside the heliosphere to Earth. The GCR flux near Earth is modulated throughout the sunspot (11-year [8]) and solar magnetic (22-year [15]) cycles. As a result, observations of this solar modulation provide important information about global properties of the heliosphere because the GCR transport is affected by the interplanetary magnetic field (IMF), magnetic turbulence, and solar wind speed. High-precision measurements of solar modulation of GCRs are available from fixed NM stations, and these are the subject of the present work. Each NM count rate is an integral measurement above a rather sharp cutoff rigidity. At low cutoff rigidity, there have been continuous measurements of solar modulation for six solar cycles [20, 23].

These detailed time series have defied a simple explanation. One popular model is the spherically symmetric force field model, in which cosmic ray spectra at Earth are characterized by a single modulation parameter, $\phi[5,13]$. Based on $\mathrm{NM}$ observations at low cutoff rigidity, empirical modulation parameters have been tabulated as a function of time [12, 24]. Furthermore, there are observed effects of the solar magnetic polarity, $A$. Guiding center drifts of the charged cosmic rays in the IMF $[14,16]$ were proposed as a mechanism to explain changes in the time profiles of NM count rates upon a solar magnetic polarity reversal [15]. Another proposed mechanism involves the effect of magnetic helicity on GCR diffusion [3]. Both effects should depend on the product $q A$. Such charge sign-dependent modulation has been observed [1, 2, 9], but charge sign and energy dependences are not yet fully resolved.

So far, most reported analyses of solar modulation based on NM time series were for relatively low cutoff rigidity; there are not many long-term time series of NM data at $P_{c}>12 \mathrm{GV}$, as shown in Figure 1. Here we express $P_{c}$ using the apparent cutoff rigidity $[4,7]$ determined using the

Figure 1: Time periods and apparent cutoff rigidities above 9.5 GV for which NM data are available. Reversals in solar magnetic polarity, near sunspot maxima, are indicated by vertical lines. Since NMs are sensitive to relativistic (positively charged) GCR ions, positive magnetic polarity $A>0$ results in $q A$-positive solar modulation, and $A<0$ results in $q A$-negative solar modulation. Among the few time series spanning a solar cycle, the NM data from Doi Inthanon, Thailand have the highest apparent cutoff rigidity (over $17 \mathrm{GV}$ ) and are the focus of this study.

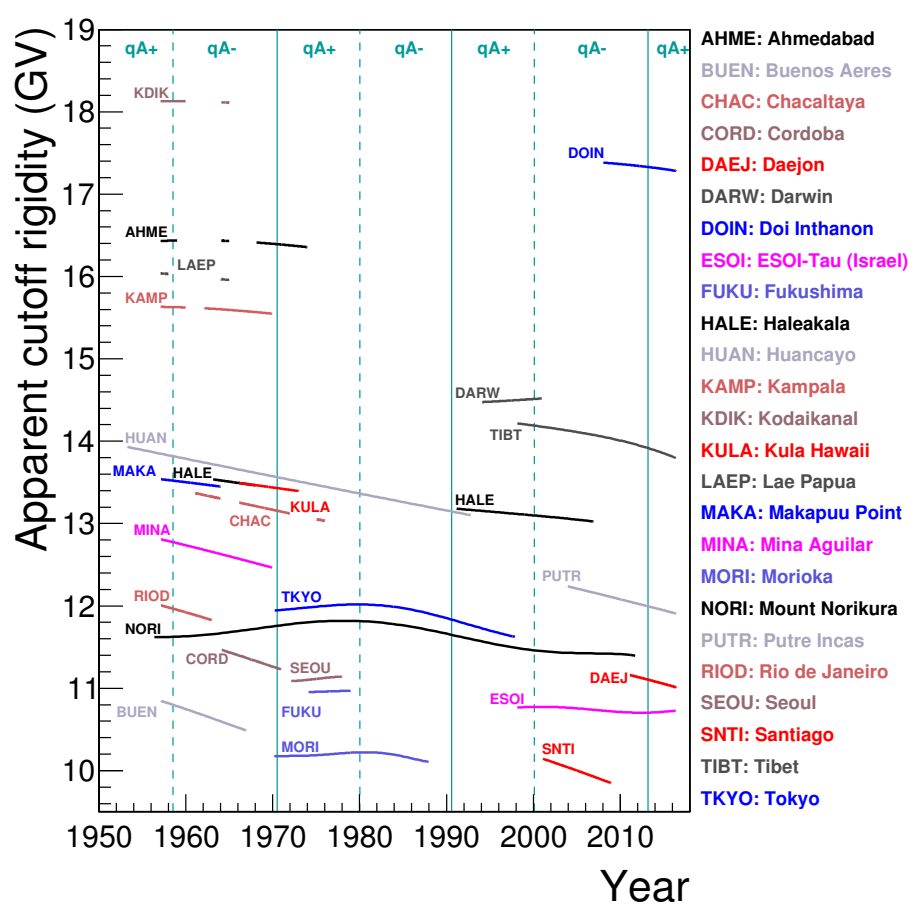


International Geomagnetic Reference Field (IGRF-12) and the methods of [17]. For NM stations worldwide with $P_{c}>9.5 \mathrm{GV}$, Figure 1 shows the time of operation and $P_{c}$. We see that there were few NM stations at high $P_{c}$ with data that spanned a solar cycle and a magnetic polarity reversal. Among these, only the Princess Sirindhorn Neutron Monitor at the summit of Doi Inthanon (DI), Thailand $\left(18.59^{\circ} \mathrm{N}, 98.49^{\circ} \mathrm{E}\right.$, altitude $\left.2560 \mathrm{~m}\right)$ had $P_{c}>17 \mathrm{GV}$, which is the highest cutoff of any NM currently in operation.

In this paper we show that the solar modulation of GCRs as observed at DI from 2007 December to 2017 January exhibited a qualitatively distinct pattern from that observed at low cutoff rigidity. We also demonstrate that a force-field model using an empirical modulation parameter $\phi(t)$ as determined from low-cutoff NM data provides a poor description of the time profile measured at DI. In other words, the spectral effects of solar modulation over roughly 1 to $17 \mathrm{GV}$ are too complex to be well described by a single modulation parameter. We compare the DI NM count rate profile with low- $P_{c}$ data from McMurdo, as well as the sunspot number, tilt angle of the heliospheric current sheet (HCS), and IMF, and we identify different patterns of solar modulation during periods of low and high tilt angle.

\section{Solar Modulation at High Cutoff Rigidity}

Neutron Monitor data preparation. PSNM is operated at DI with a complete set of 18 BP-28 $\left({ }^{10} \mathrm{BF}_{3}\right)$ neutron counter tubes with the standard NM64 configuration (18NM64). Further details are available from [22]. The present study considers monthly or Carrington (solar) Rotation (CR) averages. The count rate has been corrected for variations in atmospheric pressure. Because of important differences in meteorological conditions at this tropical location between the rainy and the dry seasons, the pressure-corrected count rates have been further corrected using the watervapor pressure at the surface. The peak-to-peak annual wave in the pressure corrected count rate is not negligible and is estimated at $\sim 0.3 \%$ ( $\sim 10 \%$ of the peak-to-peak solar modulation). More details about this correction will be presented in an article in preparation.

Neutron monitor count rates. Figure 2(a) and 2(b) present the NM count rates averaged over CRs for DI and McMurdo, respectively. From late 2007, the two NM count rates increased until a maximum in late 2009. This GCR maximum followed a very low minimum in the mean total sunspot number (Figure 2(c)) as reported by WDC-SILSO, Royal Observatory of Belgium, Brussels $^{1}$, with some delay as expected for solar effects to propagate throughout the heliosphere. After the GCR maximum, both NM count rates mostly decreased throughout 2010. Then two distinct patterns developed: the McMurdo count rate (at low cutoff rigidity) generally decreased from mid2010 until early 2015, whereas the DI count rate (at high cutoff rigidity) remained relatively stable up to mid-2014. This 4-year plateau started about 2 years before the polarity reversal at the sun observed by WSO (Figure 2(d)) and more than a year before the first polarity reversal in the north hemisphere of the Sun. We can safely assume that this plateau is not directly related to the solar polarity reversal. After removing the main Forbush decreases, some relatively large variations of the count rates at DI with a time scale of several CRs can still be observed. Those variations are also seen in the count rate at McMurdo. This period is followed by a very fast decrease of the

\footnotetext{
${ }^{1}$ See http://sidc.be/silso/home
} 

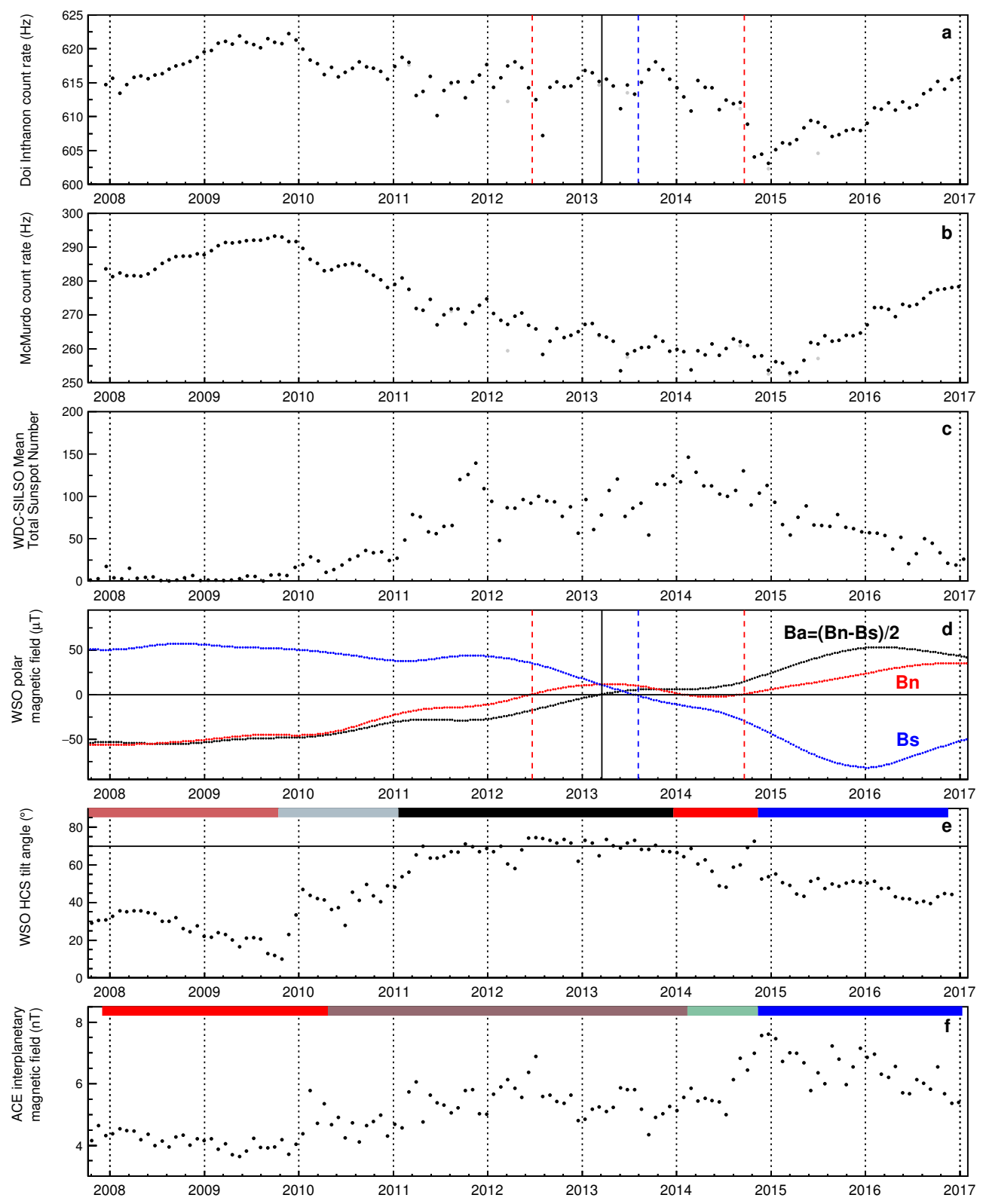

Figure 2: Time series from late 2007 to early 2017 of (a) the pressure- and water vapor-corrected NM count rate at Doi Inthanon, averaged over Carrington rotations (CRs), (b) the pressure-corrected NM count rate at McMurdo, Antarctica, averaged over CRs, (c) WDC-SILSO monthly mean total sunspot number, (d) WSO solar polar magnetic fields (traces explained in the text), (e) WSO heliospheric current sheet tilt angle, averaged over CRs, and (f) ACE-MAG interplanetary magnetic field, averaged over CRs. In (a) and (b), fluxes before and after removing periods of strong Forbush decreases are shown by gray and black points, respectively. Vertical lines in panels (a) and (d) indicate the first and last zero crossings of Bn (red, dashed lines) and the zero crossings of Bs (blue, dashed line) and Ba (black, solid line). The horizontal line in panel (e) indicates a tilt angle of $70^{\circ}$; values above this line should be considered as lower limits. The color code over panel (e) indicates time periods of distinct tilt angle levels or trends, as used in Figure 5, while another color code over panel (f) indicates time periods of distinct trends in the count rate at DI, as used in Figures 3 and 6. 
DI count rate that led to the count rate minimum at the end of 2014. The time of the decrease matched with the increase of the ratio $e^{+} / e^{-}$observed by PAMELA [1] and the end of the polarity reversal propagation through the whole heliosphere. From early 2015, the two NM count rates increased until 2017. The last large Forbush decrease was observed in June 2015 and marked the end of the sunspot maximum. The CR-averaged NM count rates at Doi Inthanon and McMurdo are also compared in Figure 3. We note that from December, 2007 (the start of our analysis period) to April, 2010 and from July, 2015 to January, 2017 (the end of our analysis period), there is a very good linear relationship between the two count rates. Note that these time periods correspond to relatively lower tilt angles, roughly speaking when the tilt angle was below about $50^{\circ}$. Such a linear relationship was also found for a ship-borne NM and the McMurdo NM during 1994-2007 [19], with the exception of the year 2000, which again was when the tilt angle was high. Note that the period of high tilt angle during the previous solar cycle (during 2000) was much shorter than during the present solar cycle (during 2010-2014). We also confirm the results of [19,21] that the slope of the linear relationship between NMs at different cutoffs was higher during the time of negative solar magnetic polarity (Figure 3(b)). This Figure also shows that the slopes were very similar during the periods of rising and falling GCR flux at Doi Inthanon during the $q A<0$ period.
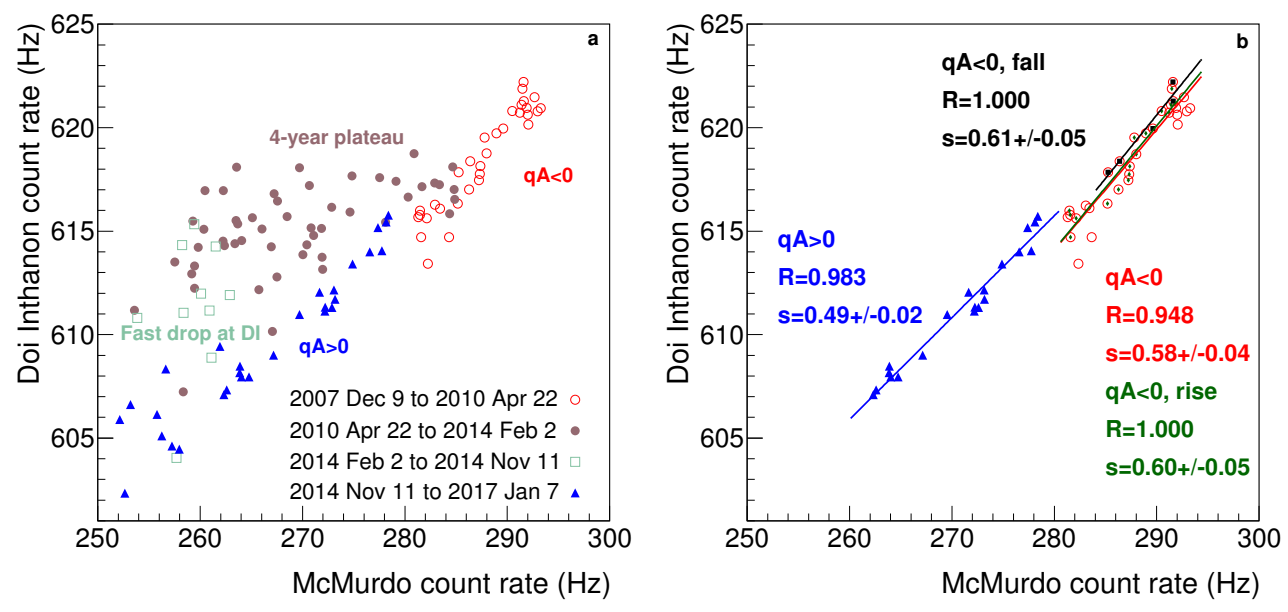

Figure 3: (a) Scatter plot of neutron monitor count rates at Doi Inthanon, Thailand (high cutoff rigidity) and McMurdo, Antarctica (low cutoff rigidity) from December, 2007 to January, 2017. (b) Same plot restricted to the earliest and latest time periods, for a low solar activity and a heliospheric current sheet tilt angle $\lesssim 50^{\circ}$; at such times the two stations' count rates have a linear relationship, and the slope depends on solar magnetic polarity as found by [19,21]. For higher tilt angle, from mid-2010 to late 2014, there is no longer a linear relationship, as DI has a distinct pattern of solar modulation from that observed at low cutoff.

Comparison with force-field model using modulation parameter determined from monitors at lower cutoff rigidity. For three NM stations, we derived the time series of the spectra of proton and helium at the top of the atmosphere from time series of the solar modulation parameter as seen by a single $\mathrm{NM}^{2}[10,11,12], \Phi_{N M}$. We then applied the simulated yield functions from [18] to determine the count rates expected at DI. The comparison of the simulated count rates with the

\footnotetext{
${ }^{2}$ Data are available at http://lpsc.in2p3.fr/crdb
} 
observations at DI are presented in Figure 4. During times of high tilt angle, the solar modulation parameter time series based on NMs at low cutoff provide a poor description of solar modulation at Doi Inthanon, at high cutoff, indicating that solar modulation over a wide rigidity range is not well described by a single empirical modulation parameter. These results are compatible with a spectral crossover at 5-6 GV.

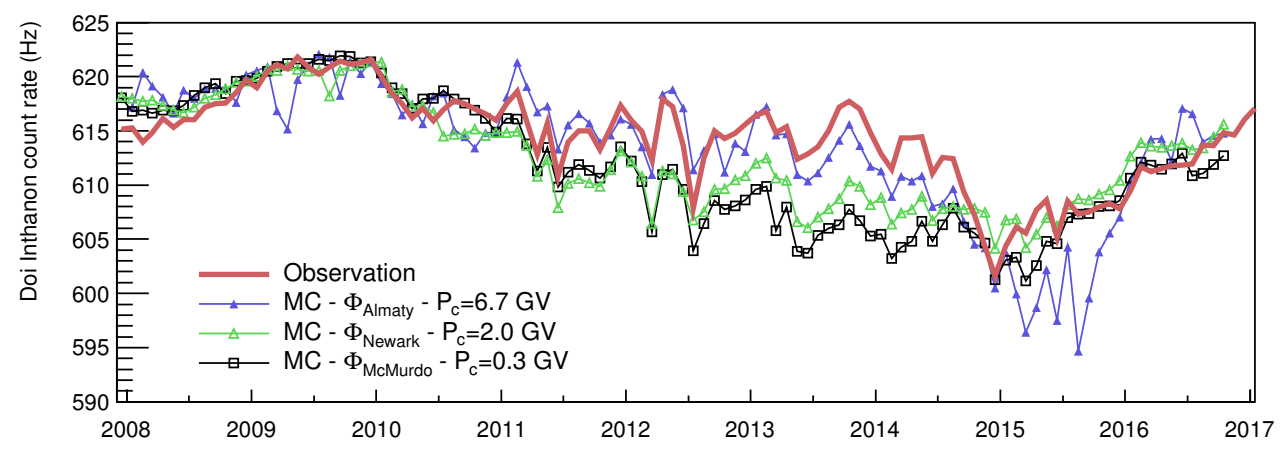

Figure 4: Comparison of the count rate at Doi Inthanon with simulated count rates estimated using the Local Interstellar Spectrum from [10,11] with the force-field approximation. The solar modulation parameters were estimated from NMs with different rigidity cutoffs, i.e., Almaty, McMurdo, and Newark with the method presented in [12]. The Monte Carlo count rates were normalized to that of PSNM at the cosmic ray maximum (December 2009).

Comparison with tilt angle of heliospheric current sheet. During $q A<0$, the excess count rate during the solar minimum coincides with a very low value of the tilt angle, $10-20^{\circ}$. This is in agreement with the calculation of [25]. Their model, which includes drift and a wavy neutral sheet, shows a dependence on the tilt angle of the solar modulation during the negative solar polarity. Small tilt angle implies a low modulation effect and thus a high count rate in comparison to that at large tilt angle. From their calculation, we can also expect a much larger dependence on the tilt angle below $30^{\circ}$ and a plateau in the modulation for $\alpha \gtrsim 50^{\circ}$. This behaviour is observed at both DI and McMurdo, respectively, in Figures 5(a) and 5(b). During $q A>0$, the count rates are less correlated with the tilt angle as expected from solar modulation models that include drifts [25].

Comparison with interplanetary magnetic field. Figure 2(f) presents the time series of the IMF during the period of interest of this work. Inverse correlation between the IMF and the count rate of a NM at low cutoff (Mount Wellington) was already observed and described in [6]. They also reported a loss of correlation in 1969, 1980 and 1990 near the periods of solar polarity reversal. In Figures 6(a) and 6(b) we compared, respectively, the count rates at DI and at McMurdo with the IMF. The loss of correlation, at low rigidity cutoff, between the count rate at McMurdo and the IMF is observed during not only the polarity reversal but during the full period of high tilt angle, i.e., the 4-year plateau (filled circles). However, at DI the correlation at high rigidity cutoff between the count rate at DI and the IMF remained during times at high tilt angle. We observed here a distinct pattern between the modulations at high and low cutoff during the period of high tilt angle and strong solar activity. This could also explain the loss of correlation between the data of the latitude surveys and McMurdo near 2000. This is consistent with an expected increase in diffusion at high 

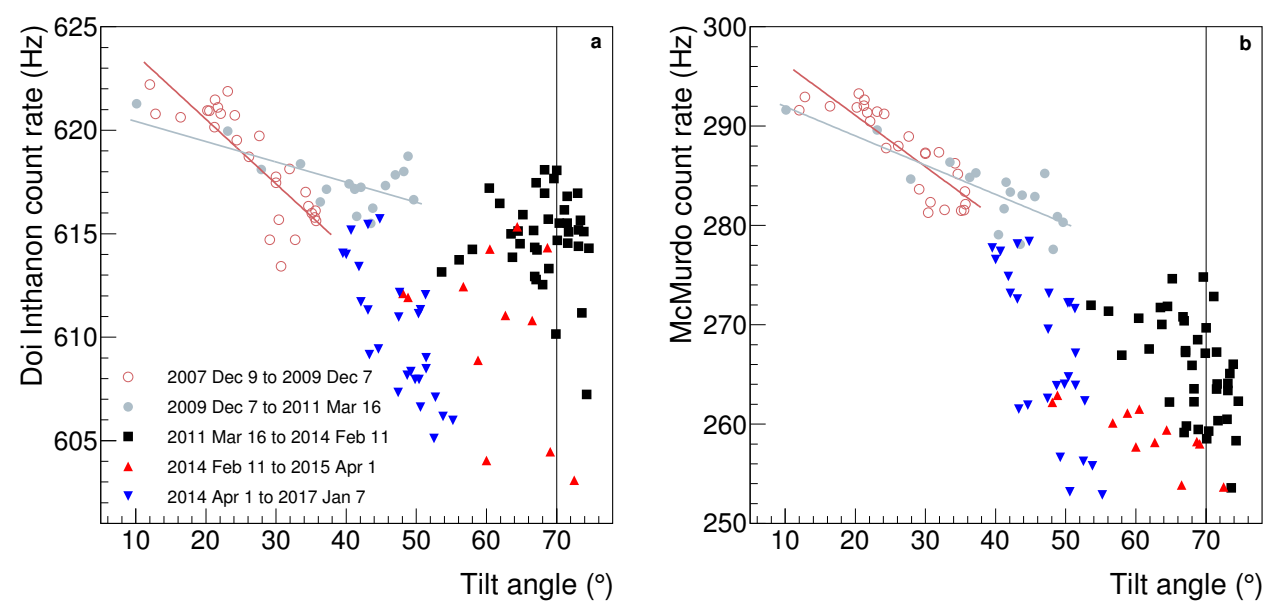

Figure 5: Correlations between the count rates (average per CR) at DI (a) and McMurdo (b) with the WSO heliospheric current sheet tilt angle, averaged over CRs. A delay of two CRs was applied to the count rates to maximize the correlation factors.
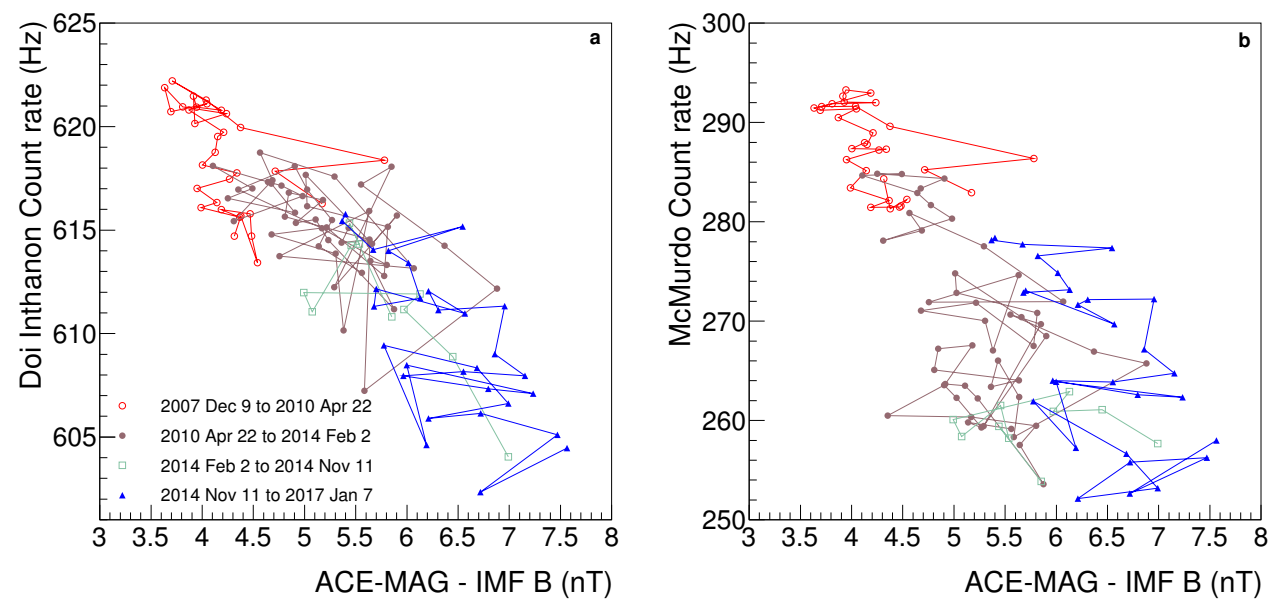

Figure 6: Correlations between the count rates (average per CR) at DI (a) and McMurdo (b) with ACE-MAG interplanetary magnetic field, averaged over CRs.

rigidity short-circuiting effects of drifts and the heliospheric current sheet for high tilt angle.

\section{Conclusions}

During the period of low solar activity, the modulation at high and low cutoffs are linearly related as found by [19]. The slope of the linear relationship depends mainly on the solar polarity. However, during the period of high solar activity, there is a distinct pattern of solar modulation at high cutoff rigidity. A plateau in the count rate at DI is observed while the count rate at McMurdo keeps decreasing. Thus a simple single modulated parameter derived from low cutoff NM data is inadequate to reproduce the modulation at high rigidity. 
Physically, at low tilt angle during the $q A<0$ polarity (2007 December - 2011 March), the tilt angle is well correlated with NM count rates at low or high rigidity, as expected by drift models. However, at high tilt angle, the DI count rate is better explained by the IMF, which is related to diffusion coefficients. Indeed the overall correlation for the entire solar modulation cycle between the DI count rate and the IMF is quite strong.

\section{Acknowledgments}

Partially supported by the Thailand Research Fund awards RTA5980003 and TRG5880173, the United States National Science Foundation awards PLR-1245939, PLR-1341562 and their predecessors.

\section{References}

[1] Adriani, O., Barbarino, G. C., Bazilevskaya, G. A., et al. 2016, PhRvL, 116, 241105

[2] Asaoka, Y., Shikaze, Y., Abe, K., et al. 2002, PhRvL, 88, 051101

[3] Bieber, J. W., \& Burger, R. A. 1990, ApJ, 348, 597

[4] Bieber, J. W., Clem, J., \& Evenson, P. 1997, in Proc. 25th ICRC (Durban), 2, 389

[5] Caballero-Lopez, R. A., \& Moraal H. 2004, JGR, 109, A01101

[6] Cane, H. V., Wibberenz, G., Richardson, I. G. \& Von Rosenvinge, T. T., 1999, GRL, 26, 5, 565-568

[7] Clem, J. M., Bieber, J. W., Evenson, P., et al. 1997, JGR, 102, 26919

[8] Forbush, S. E. 1954, JGR, 59, 525

[9] Garcia-Munoz, M., Meyer, P., Pyle, K. R., Simpson, J. A., \& Evenson, P. 1986, JGR, 91, 2858

[10] Ghelfi, A., Barao, F., Derome, L., \& Maurin, D. 2016, A\&A, 591, A94

[11] Ghelfi, A., Barao, F., Derome, L., \& Maurin, D. 2016, erratum: arXiv:1511.08650v3

[12] Ghelfi, A., Maurin, D., Cheminet, A., et al. 2017, AdSpR, in press

[13] Gleeson, L. J., \& Axford, W. I. 1968, ApJ, 154, 1011

[14] Jokipii, J. R., Levy, E. H., \& Hubbard, W. B. 1977, ApJ, 213, 861

[15] Jokipii, J. R., \& Thomas, B. 1981, ApJ, 243, 1115

[16] Levy, E. H. 1976, JGR, 81, 2082

[17] Lin, Z., Bieber, J. W., \& Evenson, P. 1995, JGR, 100, 23543

[18] Mangeard, P.-S., Ruffolo, D., Sáiz, A., Madlee, S., \& Nutaro, T. 2016a, JGRA, 121, 743

[19] Nuntiyakul, W., Evenson, P., Ruffolo, D., et al. 2014, ApJ, 795, 11

[20] Oh, S., Bieber, J. W., Evenson, P., et al. 2013, JGRA, 118, 5431

[21] Popielawska, B. 1992, P\&SS, 40, 811

[22] Ruffolo, D., Sáiz, A., Mangeard, P.-S., et al. 2016, ApJ, 817, 38

[23] Simpson, J. A. 2000, SSRv, 93, 11

[24] Usoskin, I. G., Bazilevskaya, A., \& Kovaltsov, G. A. 2011, JGR, 116, A02104

[25] Webber, W. R., Potgieter, M. S., \& Burger, R. A. 1990, ApJ, 349, 634 